\title{
Analyte-Responsive Hydrogels: Intelligent Materials for Biosensing and Drug Delivery
}

\author{
Heidi R. Culver ${ }^{\dagger, \ddagger}$, John R. Clegg ${ }^{\dagger, \ddagger}$, and Nicholas A. Peppas ${ }^{\star}, \dagger, \ddagger, \S, \|, \perp$ \\ †Institute for Biomaterials, Drug Delivery, and Regenerative Medicine, C0800, The University of \\ Texas at Austin, Austin, Texas, 78712, United States \\ ‡Department of Biomedical Engineering, C0800, The University of Texas at Austin, Austin, Texas \\ 78712, United States \\ \$McKetta Department of Chemical Engineering, C0400, The University of Texas at Austin, Austin, \\ Texas 78712, United States \\ "Department of Surgery and Perioperative Care, Dell Medical School, The University of Texas at \\ Austin, Austin, Texas 78712, United States \\ ${ }^{\perp}$ College of Pharmacy, A1900, The University of Texas at Austin, Austin, Texas 78712, United \\ States
}

\section{CONSPECTUS:}

Nature has mastered the art of molecular recognition. For example, using synergistic non-covalent interactions, proteins can distinguish between molecules and bind a partner with incredible affinity and specificity. Scientists have developed, and continue to develop, techniques to investigate and better understand molecular recognition. As a consequence, analyte-responsive hydrogels that mimic these recognitive processes have emerged as a class of intelligent materials. These materials are unique not only in the type of analyte to which they respond but also in how molecular recognition is achieved and how the hydrogel responds to the analyte. Traditional intelligent hydrogels can respond to environmental cues such as $\mathrm{pH}$, temperature, and ionic strength. The functional monomers used to make these hydrogels can be varied to achieve responsive behavior. For analyte-responsive hydrogels, molecular recognition can also be achieved by incorporating biomolecules with inherent molecular recognition properties (e.g., nucleic acids, peptides, enzymes, etc.) into the polymer network. Furthermore, in addition to typical swelling/syneresis responses, these materials exhibit unique responsive behaviors, such as gel assembly or disassembly, upon interaction with the target analyte. With the diverse tools available for molecular recognition and the ability to generate unique responsive behaviors, analyte-responsive hydrogels have found great utility in a wide range of applications.

In this Account, we discuss strategies for making four different classes of analyte-responsive hydrogels, specifically, nonimprinted, molecularly imprinted, biomolecule-containing, and enzymatically responsive hydrogels. Then we explore how these materials have been incorporated

*Corresponding Author: peppas@che.utexas.edu.

Notes

The authors declare no competing financial interest. 
into sensors and drug delivery systems, highlighting examples that demonstrate the versatility of these materials. For example, in addition to the molecular recognition properties of analyteresponsive hydrogels, the physicochemical changes that are induced upon analyte binding can be exploited to generate a detectable signal for sensing applications. As research in this area has grown, a number of creative approaches for improving the selectivity and sensitivity (i.e., detection limit) of these sensors have emerged. For applications in drug delivery systems, therapeutic release can be triggered by competitive molecular interactions or physicochemical changes in the network. Additionally, including degradable units within the network can enable sustained and responsive therapeutic release. Several exciting examples exploiting the analyteresponsive behavior of hydrogels for the treatment of cancer, diabetes, and irritable bowel syndrome are discussed in detail. We expect that creative and combinatorial approaches used in the design of analyte-responsive hydrogels will continue to yield materials with great potential in the fields of sensing and drug delivery.

\section{Graphical Abstract}

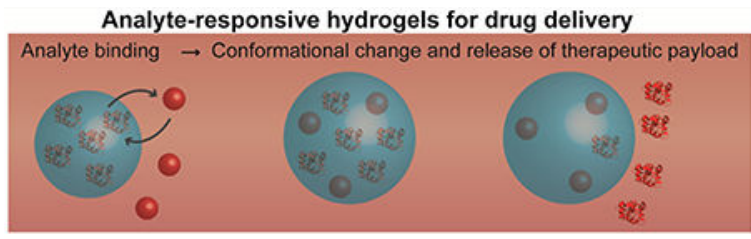

Analyte-responsive hydrogels for sensing

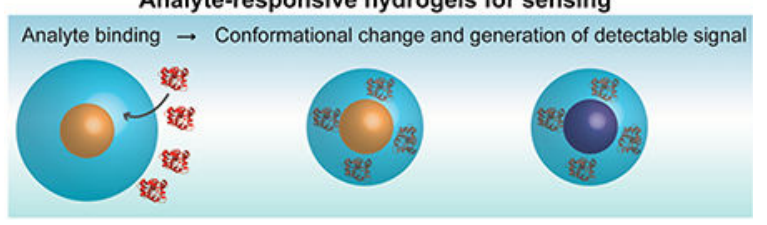

\section{INTRODUCTION}

Stimuli-responsive hydrogels have been investigated for many decades. By harnessing the environmental sensitivity of crosslinked polymer networks, scientists can control and manipulate the behavior and properties of these materials for many applications. In general, changes in hydrogel properties are due to interactions between pendant functional groups, the solvent, and ions. ${ }^{1}$ Beyond these interactions, analyte-responsive hydrogels uniquely interact with, and respond to, specific complex molecules (i.e., analytes). The development of analyteresponsive materials requires interaction with a specific molecule to cause an event, which can range from high-affinity binding to degradation of the hydrogel. To generate such materials, scientists have looked to nature for inspiration. In particular, a variety of analyte-responsive hydrogels have been developed to emulate molecular recognition events that exist in nature, motivated by our understanding of the non-covalent interactions that govern strong and specific molecular adsorption. ${ }^{2}$

The versatility of analyte-responsive hydrogels, in terms of how molecular recognition is achieved, analyte diversity, responsive behaviors, and biomedical applications, is illustrated in Figure 1. First, the figure demonstrates that there are many ways to achieve molecular 
recognition in the hydrogel. In traditional polymer systems, molecular recognition is achieved by incorporating functional monomers that form favorable interactions with an analyte of interest. To better control the incorporation of these functional groups and improve the affinity for the analyte, molecular imprinting strategies can be used. Alternatively, biomolecules with well-characterized affinity for an analyte can be conjugated to the polymer to achieve recognition and impart responsive properties.

Figure 1 also shows that these systems can be engineered to respond to a variety of analytes ranging from toxic heavy metal ions (e.g., $\mathrm{Hg}^{2+}$ and $\mathrm{Pb}^{2+}$ ) to macromolecules and cells. Additionally, the figure emphasizes the various ways that hydrogels respond to analytes. While the responsive behavior may simply be binding the analyte of interest, in most cases the molecular recognition event is concomitant with a conformational response. For example, interaction with the template can lead to a change in the microenvironment within the hydrogel (e.g., pH, net charge, etc.) that leads to swelling or syneresis (i.e., deswelling). Complementary DNA can form cross-links that lead to hydrogel assembly, while enzymatically cleavable peptide cross-linkers disassemble following interaction with their target enzyme.

The last aim of Figure 1 is to illustrate that because of these degrees of tunability, the applications of analyte-responsive hydrogel materials are creative and widespread. The primary focus of this Account is to discuss the unique ways in which analyte-responsive hydrogels are being applied in sensors and drug delivery systems. For example, these materials can be utilized to detect trace amounts of heavy metal ions in order to protect the health of humans and other animals. Furthermore, alternative analyte-responsive hydrogels capable of capturing or releasing small molecules or macromolecules have great utility for both sensing and drug delivery applications. Mechanistically, binding a target analyte can lead to molecular reorientation or displacement events that are useful for producing detectable signals or releasing therapeutic payloads. In the following sections, we briefly introduce four classes of analyte-responsive hydrogels before providing a more in-depth discussion of their applications in sensing and drug delivery.

\section{CLASSES OF ANALYTE-SENSITIVE MATERIALS}

Analyte-responsive hydrogels can be made from a variety of materials with a range of complexity. All of the gels require a molecular recognition element that initiates the responsive behavior. In this section, we describe the different approaches that have been used to achieve molecular recognition in analyteresponsive hydrogels.

\section{A. Non-imprinted Recognitive Hydrogels}

A very basic form of recognition is related to size exclusion, which is achieved by progressive addition of molecular barriers such as cross-links, tie points, and crystallites. These barriers allow molecules below a specific molecular weight to diffuse through the network's pores, while larger species are excluded. In addition, monomers that form favorable non-covalent interactions with an analyte of interest can be incorporated into the hydrogel. As an example, Luchini and colleagues developed Nisopropylacrylamide nanoparticles as affinity receptors for protein binding. They first varied the extent of cross- 
linking to exclude albumin from the nanoparticles while allowing capture of lowermolecular-weight, less abundant proteins. Then they included acrylic acid as a comonomer and "affinity bait" in order to preferentially capture high-isoelectric-point proteins and peptides. ${ }^{3}$ Alternatively, natural polymers such as hyaluronic acid, which inherently present well-ordered functional groups, can also be used to develop analyte-recognitive systems. ${ }^{4}$

In order to achieve even higher affinity and specificity, multifunctional molecules can be incorporated into hydrogels during polymerization or through postsynthetic modification. For example, Schrader and colleagues designed a variety of monomers that have high affinity for specific amino acids. ${ }^{5}$ In an alternative strategy, the Luchini group used zerolength crosslinker amidation reactions to incorporate high-affinity bait molecules, specifically amine-containing dyes, into their acrylic acid-containing hydrogels. These particles were capable of sequestering a variety of protein biomarkers from complex biological fluids. ${ }^{6,7}$ These examples demonstrate how researchers have designed polymers to achieve various levels of recognition specificity.

\section{B. Molecularly Imprinted Synthetic Hydrogels}

Molecularly imprinted polymers (MIPs), which were originally fabricated for chromatography applications, are cross-linked networks that possess selective affinity for template molecules. MIPs are synthesized by incubating template analytes with functional monomers prior to polymerization, allowing the formation of reversible-covalent or noncovalent interactions. Following polymerization, a series of solvents are used to extract the entrapped template and isolate the purified MIP, ideally without modifying the polymer composition or structure. Through the synergistic effects of favorable intermolecular interactions and shape complementarity, the resulting MIP systems possess selective affinity for a variety of analytes. 8,9

MIPs can be classified by template selection, imprint location, polymerization method, and polymer macroscopic structure. Traditional imprinting utilizes small molecules, proteins, or cells as templates, while epitope imprinting approaches use a moiety or peptide instead of the entire molecule. The whole molecule or epitope can be located throughout the polymerization solution, resulting in bulk imprinting, or restricted to the interface of the polymerization solution, resulting in surface imprinting. MIPs have been synthesized using free radical and living polymerization, each of which presents unique advantages depending on the reaction conditions (e.g., time frame, solvent, temperature) that are best suited for the template. MIPs have been fabricated in particles and films, on the nano- and microscale, for a variety of medical applications.

\section{C. Biomolecule-Containing Hydrogels}

Beyond generating molecular recognition elements in hydrogels through purely synthetic approaches, recognitive biomolecules can be conjugated to or encapsulated in hydrogel networks. In one strategy, the biomolecule is acrylated and included during the polymerization, ${ }^{10}$ which works well for biomolecules that are soluble and stable in the reaction medium. Alternatively, postsynthetic modifications can decorate hydrogels with recognitive biomolecules. These strategies are particularly useful for proteins, which have 
delicate tertiary structures and can be denatured under harsh polymerization conditions. In order to link the biomolecule to the hydrogel, both the polymer and the biomolecule must have functional groups available for bioconjugation. ${ }^{11}$

In some cases, it is desirable that the biomolecule have some freedom to move within the hydrogel's pores. In these cases, rather than covalently linking the biomolecule to the polymer, the biomolecule is encapsulated in the hydrogel either during or after gelation. The strategy chosen for loading recognitive biomolecules into hydrogels depends on the biomolecule and application. Hydrogels that assemble under gentle reaction conditions are convenient for loading delicate biomolecules as the gel forms. ${ }^{12,13}$ For polymerizations that require potentially denaturing reaction conditions (e.g., heat, extreme $\mathrm{pH}$, organic solvents, etc.) delicate biomolecules can be loaded into the preformed network via equilibrium partitioning. ${ }^{14}$

\section{D. Enzymatically Responsive Hydrogels}

In addition to the incorporation of enzymes into hydrogels through covalent linkage or encapsulation, enzymes can interact directly with hydrogels that employ peptide-substrate linkers. A linker can be used to couple a therapeutic agent directly to a polymeric carrier such that enzymatic degradation of the linker releases the drug. Alternatively, peptide crosslinking agents can be used to form network polymers, where enzymes systematically cleave the linkers, decrease effective cross-linking, increase the polymer-swelling ratio, and increase molecular permeability. Typical chemical methods used to achieve cross-linking of peptide-polymer hybrids include thiol-norbornene click reactions ${ }^{15}$ with oligopeptidependant cysteines or carbodiimide-mediated coupling of pendant lysines or aspartic acids to the corresponding polymeric carboxylic acids or amines. ${ }^{14}$ Peptide linkers have been designed for tunable degradation by a number of native enzymes, including matrix metalloproteinase, ${ }^{15}$ trypsin, ${ }^{14}$ and elastase. ${ }^{16}$

\section{SENSING APPLICATIONS OF ANALYTE-RESPONSIVE HYDROGELS}

Sensors require an element for molecular recognition and an element for signal transduction. More specifically, a binding event between a receptor and its ligand must result in a detectable signal, most commonly an optical or electrochemical output. Ideal sensors are inexpensive, environmentally stable, and have low detection limits (i.e., high sensitivity). The recent examples discussed below show how analyte-responsive hydrogels have been used in sensing applications.

\section{A. Recognitive and Molecularly Imprinted Polymers for Sensing}

As described above, molecular recognition in synthetic hydrogels can be achieved through incorporation of bait molecules and/or molecular imprinting. These materials are inexpensive and environmentally robust, making them attractive for use in sensors. The responsive behavior of hydrogels upon analyte binding, particularly swelling or deswelling, is commonly exploited for signal transduction. Upon hydrogel swelling or deswelling, two changes are obvious: a change in volume and a change in refractive index. By incorporation 
of materials that are sensitive to such changes, signal transduction is achieved upon analyte binding.

For example, the diffraction wavelength, and consequently the apparent color, of photonic crystals depends on the lattice spacing of the colloidal array. Swelling or deswelling initiated by analyte binding increases or decreases the lattice spacing of embedded colloidal crystal arrays, respectively, resulting in a change in color. Asher and colleagues applied this concept to detect a variety of analytes. ${ }^{17}$ The ease of incorporating functional groups into hydrogels during the polymerization enabled them to detect many analytes, including metal cations $\left(\mathrm{Pb}^{2+}\right),{ }^{18}$ small molecules (glucose), ${ }^{19}$ and proteins (concanavalin A) (Figure 2) ${ }^{20}$ In a similar concept, hydrogels can be synthesized around a close-packed array of spherical particles that are then extracted, generating an array of spherical voids called an "inverse opal". ${ }^{21}$ Compared with traditional photonic crystals (i.e., opals), inverse opals have large pores that facilitate analyte diffusion into the sensor, which is particularly useful for macromolecule detection. ${ }^{22}$ The concept of inverse opals has been combined with molecular imprinting strategies to develop sensors for analytes including tetracycline ${ }^{23}$ and bovine serum albumin. ${ }^{24}$

As mentioned above, analyte-induced swelling or deswelling also results in a change in the hydrogel refractive index due to water imbibition or release, respectively. This change in refractive index can be detected using surface plasmon resonance (SPR)based sensing techniques. In particular, gold nanomaterials exhibit localized SPR (LSPR), which results in strong absorption of light at a particular wavelength. The wavelength at which nanomaterials absorb depends on their size, shape, and, most importantly, local refractive index. Mesch et al. ${ }^{25}$ took advantage of this sensitivity to the refractive index to develop a glucose sensor. They combined gold nanoantennas with a phenylboronic acid-containing hydrogel that reversibly swelled upon glucose binding. As glucose bound and the hydrogel swelled, the LSPR wavelength of the nanoantennas blue-shifted because of the decrease in refractive index. Using this sensing strategy, they were able to detect physiological changes in glucose concentration.

In addition to being used directly in sensors for molecular recognition and/or signal transduction, recognitive polymers have been used as tools for isolating or concentrating target molecules prior to detection. For example, Derazshamshir et al. ${ }^{26}$ developed imprinted cryogels to deplete hemoglobin, an abundant protein in human serum. Such imprinted hydrogels can be used to deplete certain proteins and consequently improve the detection of less abundant biomarkers. In an alternative approach, Magni et al. ${ }^{7}$ used the "Nanotrap" technology developed by their laboratory to concentrate the outer-surface protein A of Borrelia, which is a biomarker for lyme borreliosis. This preconcentration step was used to enhance the sensitivity of immunoassays.

\section{B. Biomolecule-Containing Hydrogels for Sensing}

Recognitive and molecularly imprinted polymers are particularly useful for low-cost sensing applications and cases where analytes do not have a known binding partner. Biomoleculecontaining hydrogels, on the other hand, are typically more expensive and utilize biomolecules with well-established molecular recognition properties. For these materials, 
the main area of exploration is the approach for signal transduction, with the goal being to improve the detection limit of the sensor. In one strategy, the Liu group made monolithic DNA-containing hydrogels that enabled visual detection of target DNA using colloidal gold. More specifically, the intensity of the red color from the gold nanoparticles (i.e., the optical density) was sensitive to the target DNA concentration down to $100 \mathrm{pM}$. To improve the detection limit, they took advantage of colloidal gold's ability to reduce silver. This strategy lowered the detection limit to $1 \mathrm{pM}$, based on visual observation of a black color in the hydrogel as a result of silver reduction. ${ }^{27}$ The same group fabricated hydrogel microparticles that contained a high-affinity aptamer for mercury $\left(\mathrm{Hg}^{2+}\right)$ and employed a fluorescencebased detection strategy. The use of aptamer-containing hydrogel microparticles enabled faster detection ( $2 \mathrm{~min}$ vs $60 \mathrm{~min}$ for monolithic gels) and improved the detection limit relative to free aptamers (10 vs $500 \mathrm{nM}$ ). Furthermore, they showed that solutions containing the gel microparticles could be spotted on a glass slide, dried, and rehydrated to generate a microarray for mercury detection. ${ }^{28}$

Several groups have used acrylated or methacrylated aptamers as macromonomers for molecular imprinting. In order to maintain the low-cost advantage of imprinting, Zhang and $\mathrm{Liu}^{10}$ used two fragments of the adenosine aptamer, which are less expensive than the fulllength aptamer. By polymerizing the methacrylated aptamer fragments in the presence of adenosine, they immobilized the two fragments in close proximity so that the adenosine -aptamer complex could later reform, causing fluorescence quenching. Compared with nonimprinted polymers synthesized with the aptamer fragments, imprinting significantly improved the sensitivity of their assay.

Beyond exploitation of biomolecules for their inherent molecular recognition characteristics, biomolecules are also useful tools for self-assembly and can enable accurate and consistent spacing of recognition ligands. For example, King et al. ${ }^{29}$ used a self-assembling octapeptide to precisely incorporate an oligonucleotide recognition motif. Self-assembly of oligonucleotide-modified and unmodified peptides resulted in wellordered presentation of the recognition motifs on the peptide- gel surface. Upon binding, the fluorescence resonance enery transfer (FRET) pair on either end of the target DNA separated, resulting in a fast and significant increase in fluorescence signal with a detection limit of $22 \mathrm{pM}$. This strategy could be applied to detect other analytes for which aptamers exist.

\section{C. Enzyme-Containing and Enzymatically Responsive Hydrogels for Sensing}

Hydrogels that contain or respond to enzymes are a particularly useful subset of analyteresponsive materials for sensing applications. Many common assays, such as enzyme-linked immunosorbent assays (ELISAs), rely on enzyme-substrate interactions to produce a detectable signal, such as the generation of a colored product. Reacting or housing enzymes within an analyte-responsive hydrogel makes it possible to generate a conformational change and detectable signal upon addition of the analyte. Furthermore, localization of the enzymes within the small volume of the hydrogel enables high local concentrations of the enzyme and, in turn, sensitive detection.

For example, Yan et al. ${ }^{12}$ encapsulated an enzyme, glucoamylase, inside an aptamer-crosslinked hydrogel. Their aptamer cross-linking scheme happened spontaneously upon addition 
of the aptamer and did not require harsh polymerization conditions that could denature the enzyme. Upon introduction of the aptamer's target, the gel disassembled and released the glucoamylase into solution, where its substrate, amylose, was located. The interaction between glucoamylase and amylose resulted in the production of glucose, which could easily be detected using a personal glucometer (Figure 3). The beauty of this strategy is that the system can easily be tailored for any nonglucose analyte for which an aptamer exists simply by changing the aptamer used for cross-linking.

Using another enzyme-encapsulating strategy, Kiyonaka et al. ${ }^{13}$ developed a synthetic material that self-assembles into fibers possessing nanoscale hydrophilic and hydrophobic domains. The aqueous cavities were used to house the enzyme lysylendopeptidase (LEP). In their sensor, a hydrophilic peptide substrate for LEP was conjugated to dansyl, a hydrophobic fluorescent dye. Upon cleavage of the peptide from dansyl by LEP, dansyl partitioned into the hydrophobic domains of the hydrogel fibers, where its fluorescence intensity significantly increased and its emission maximum shifted from 545 to $508 \mathrm{~nm}$.

The conformational changes that occur in some cases of enzyme-substrate binding present another exciting mechanism through which enzymes can provide analyte responsiveness. For example, Yuan et al. ${ }^{30}$ incorporated adenylate kinase as a crosslinker in their hydrogel network. Upon binding its substrate, ATP, adenylate kinase undergoes a nanometer-scale conformational change. With many adenylate kinase molecules simultaneously undergoing these nanoscale changes, it was possible to detect macroscopic changes in the hydrogel volume. This design could be applied using a variety of enzymes that undergo conformational changes upon substrate binding.

\section{ANALYTE CAPTURE AND RELEASE FORTHERAPEUTIC APPLICATIONS}

Analyte-responsive polymers have been employed in a variety of controlled or targeted drug delivery systems. These intelligent systems can be characterized by the network degradation, or lack thereof, and the nature of therapeutic release (Figure 4). Recent work in the development of analyte-responsive materials shows they can be used for the delivery of small-molecule and protein therapeutics.

\section{A. Recognitive and Molecularly Imprinted Polymers for Therapeutic Agent Delivery}

Hydrogels themselves, in the absence of advanced fabrication, are capable of molecular interaction and recognition. For example, while hyaluronic acid is a common biopolymer utilized in the development of natural materials, it is also a ligand for the CD44 receptor. Consequently, nanoparticles composed of, or functionalized with, hyaluronic acid can target CD44+ tumors such as glioblastoma, melanoma, ovarian cancer, breast cancer, and lung cancer. ${ }^{4}$ Comparatively, synthetic polymers are capable of favorable electrostatic interactions, hydrophobic contacts, and hydrogen bonding with either biomarkers or therapeutics. With novel and creative consideration, these interactions can be utilized to enhance drug loading and release kinetics under known physiological conditions. 
Synergistically, biomolecule- material interactions in vivo can promptly extract toxic molecules from the bloodstream ${ }^{31}$ or trigger the release of loaded drugs. ${ }^{32}$

Molecular imprinting has been utilized in numerous studies to elevate the affinity of intelligent networks for specific therapeutics. With increasing polymer-drug affinity, drug release is sustained for a greater time. Some of the original work in this area was conducted on molecularly imprinted contact lenses in an effort to extend the duration of ocular drug delivery. For example, Hiratani et al. ${ }^{33}$ synthesized timolol-imprinted lenses that enabled sustained ocular delivery to rabbits for 2-3 times the duration of non-imprinted controls. Similarly, AlvarezLorenzo et al. ${ }^{34}$ imprinted norfloxacin and achieved a 200 -fold increase in norfloxacin loading by optimizing the polymer acrylic acid content. The imprinted gels sustained norfloxacin delivery in lachrymal fluid beyond $24 \mathrm{~h}$, a significant improvement over nonimprinted controls. More recently, White et al. ${ }^{35}$ expanded upon molecularly imprinted soft contact lens technology to deliver a higher-molecular-weight therapeutic (120 kDa hydroxypropyl methylcellulose) for a greater duration (60 days). Furthermore, MIPs have been employed to obtain more ideal release kinetics from existing polymeric systems for macromolecular therapeutics. The majority of these studies have characterized the imprinting, loading, and release of model therapeutics such as lysozyme, yielding promising release kinetics. ${ }^{36}$

In contrast to utilizing therapeutic agents as imprinting templates, researchers can imprint biomarkers that facilitate targeted drug release. These systems utilize surface imprinting of whole proteins or epitopes to achieve material targeting while encapsulating or imbibing small-molecule drugs within the polymer bulk. Recently, Zhang et al. ${ }^{37}$ utilized an emulsion polymerization with epitope imprinting of the $\mathrm{p} 32$ receptor to actively target breast cancer xenografts (Figure 5). Molecular imprinting improved the cellular uptake of the drugcontaining nanogels and cytoplasmic delivery of the photosensitizer, methylene blue. Similarly, Shen et al. ${ }^{38}$ employed a Pickering emulsion polymerization to form bacteriaimprinted chitosan beads. Bacteria naturally associated at the water-oil interface, and acrylate functionalization enabled chemical cross-linking. Consequently, the surfaceimprinted chitosan networks were able to recognize specific bacterial strains.

\section{B. Biomolecule-Containing Hydrogels for Therapeutic Agent Delivery}

An alternate strategy for enhancing polymer-drug affinity for desired release behavior is to modify an otherwise nonspecific polymer with targeting ligands. For example, Soontornworajit et al. ${ }^{39}$ achieved sustained-delivery behavior by conjugating DNA aptamers with varying affinity for anti-platelet-derived growth factor-BB (anti-PDGF-BB) to polyacrylamide hydrogels. While the native hydrogels without aptamers released approximately $80 \%$ of loaded PDGF-BB within $48 \mathrm{~h}$, the aptamer-hydrogel conjugates released significantly smaller proportions of their payload and sustained release for 6 days. Alternatively, Battig et al. ${ }^{40}$ conjugated aptamers with specificity for PDGF-BB and vascular endothelial growth factor (VEGF) onto the surface of polystyrene nanoparticles contained within otherwise inert hydrogels. They optimized the aptamer length with a 16nucleotide strand and achieved specific delivery of VEGF and PDGF-BB in response to the aptamers' complementary DNA. 
Furthermore, biomolecules contained within hydrogel networks can be used as physical cross-links. In one of the first examples of this strategy, Miyata et al. ${ }^{41}$ developed hydrogels cross-linked by specific interactions between rabbit immunoglobulin $\mathrm{G}(\mathrm{IgG})$ and goat antirabbit IgG. The cross-linking of this gel, as evidenced through equilibrium swelling, was reduced in the presence of soluble rabbit $\operatorname{IgG}$ in a concentration-dependent manner, validating a competitive mechanism.

\section{C. Enzyme-Containing or Enzymatically Responsive Hydrogels for Therapeutic Agent Delivery}

Researchers can conjugate bioactive enzymes to synthetic or natural hydrogel networks, which interact directly with biomarkers in vivo and trigger therapeutic release through a physical change in the polymeric network. To utilize this strategy for glucose-responsive insulin delivery, our group immobilized glucose oxidase and catalase within microgels formed by suspension free radical polymerization. In glucose-containing buffers, glucose oxidase metabolized glucose into gluconic acid, resulting in a decrease in $\mathrm{pH}$, protonation of the tertiary amines, and, consequently, swelling of the polymeric network. Catalase closed the loop by converting the hydrogen peroxide byproduct to water and oxygen. ${ }^{42}$ Marek and Peppas ${ }^{43}$ optimized the formulation and cross-linking of this microparticle system to achieve glucose-triggered insulin release.

Gu et al. ${ }^{44}$ utilized a similar enzyme-containing system for glucose-responsive insulin delivery. Glucose-responsive nanonetworks composed of chitosan and ethoxypropenemodified dextran, containing glucose oxidase and catalase, were prepared by a double emulsion solvent evaporation method. Metabolism of glucose and the subsequent decline in $\mathrm{pH}$ catalyzed hydrolysis of the modified dextran, dissociating the nanonetworks and releasing an insulin payload. This system maintained normoglycemic levels in diabetic mice for 9-15 days with a single injection, compared with 1 day for a comparable insulin injection (Figure 6). This system has been optimized for delivery by both traditional injection and microneedle patches.

Alternatively, enzymes that exist naturally in physiological environments can interact with and modify responsive hydrogels. For example, Knipe et al. ${ }^{14}$ developed a microgel system crosslinked with a trypsin-labile oligopeptide linker. This system was stable and collapsed in acidic, trypsin-poor gastric conditions but was swollen and prone to programmed degradation in trypsinrich, neutral intestinal conditions, enabling the oral delivery of insulin. This system was subsequently modified for delivery of cationic nanoparticles associated with siRNA for TNF-a knockdown to treat inflammatory bowel diseases. ${ }^{45}$ Enzymeresponsive nanogels have also been developed with peptide linkers that are cleaved by caspase- 3 for intracellular delivery to, and induced apoptosis of, HeLa cells. ${ }^{46}$

\section{D. Polymer Modification for Molecular Recognition in Targeted Therapeutic Agent Delivery}

Conjugation of targeting ligands, such as antibodies, aptamers, or peptides, to intelligent biomaterials is a popular approach to achieve targeted drug delivery. The conjugation of ligands to drug delivery vehicles with subsequent targeting of diseased tissues is an 
extensive area of research and is the sole topic of numerous reviews. ${ }^{47-49}$ The purpose of this Account was to highlight recent progress in the field of analyte-responsive materials, but we would be remiss if we did not highlight the progress and future opportunities in coupling of analyteresponsive materials with ligand-targeting strategies for finetuned control of therapeutic retention, localization, and release.

\section{CONCLUSIONS AND OUTLOOK}

In this Account, we have described the various classes of analyteresponsive hydrogels and reviewed recent applications of these materials to highlight the versatility of this field. For biosensors, improved sensitivity and selectivity along with the potential for low production costs will make analyte-responsive hydrogels important diagnostic tools in this era of valuebased healthcare. Furthermore, controlled or targeted drug release from these hydrogels enables attractive platforms for new drug administration strategies and the reduction of offtarget side effects. We are confident that next-generation sensing and drug delivery platforms will emerge as researchers continue to utilize creative and combinatorial approaches for the design and application of analyte-responsive hydrogels.

\section{Biographies}

Heidi Culver received her B.S. degree in Materials Science and Engineering from The Johns Hopkins University in 2012. She is an NSF Graduate Research Fellow and Harrington Dissertation Fellow pursuing her Ph.D. in Biomedical Engineering at UT Austin. She is investigating recognitive polymer-nanomaterial composites for biosensing applications.

John Clegg received his B.S. degree in Biomedical Engineering from the University of South Carolina in 2014. He is a Ph.D. candidate in Biomedical Engineering and an NSF Graduate Research Fellow at UT Austin investigating synthetic recognitive polymers for drug delivery applications.

Nicholas A. Peppas is the Cockrell Family Regents Chair in Engineering \#6 and Professor of Biomedical Engineering, Chemical Engineering, Surgery, and Pharmacy at UT Austin. $\mathrm{He}$ is a member of the National Academy of Engineering, the National Academy of Medicine, the National Academy of Inventors, the National Academy of Pharmacy of France, the Royal Academy of Spain, and the Academy of Athens. He received his D.Eng. degree from the National Technical University of Athens, Greece, in 1971 and his Sc.D. from MIT in 1973, both in Chemical Engineering.

\section{口 REFERENCES}

(1). Peppas NA; Huang Y; Torres-Lugo M; Ward JH; Zhang J Physicochemical Foundations and Structural Design of Hydrogels in Medicine and Biology. Annu. Rev. Biomed. Eng 2000, 2 (1), 9-29. [PubMed: 11701505]

2). Peppas NA; Huang Y Polymers and Gels as Molecular Recognition Agents. Pharm. Res 2002, 19 (5), 578-587. [PubMed: 12069158]

3). Luchini A; Geho DH; Bishop B; Tran D; Xia C; Dufour RL; Jones CD; Espina V; Patanarut A; Zhou W; Ross MM; Tessitore A; Petricoin EF; Liotta LA Smart Hydrogel Particles: Biomarker 
Harvesting: One-Step Affinity Purification, Size Exclusion, and Protection against Degradation. Nano Lett. 2008, 8 (1), 350-361. [PubMed: 18076201]

4). Yu M; Jambhrunkar S; Thorn P; Chen J; Gu W; Yu C Hyaluronic Acid Modified Mesoporous Silica Nanoparticles for Targeted Drug Delivery to CD44-Overexpressing Cancer Cells. Nanoscale 2013, 5 (1), 178-183. [PubMed: 23076766]

5). Latza P; Gilles P; Schaller T; Schrader T Affinity Polymers Tailored for the Protein A Binding Site of Immunoglobulin G Proteins. Chem. - Eur. J 2014, 20 (36), 11479-11487. [PubMed: 25059560]

6). Tamburro D; Fredolini C; Espina V; Douglas TA; Ranganathan A; Ilag L; Zhou W; Russo P; Espina BH; Muto G; Petricoin EF; Liotta LA; Luchini A Multifunctional Core-Shell Nanoparticles: Discovery of Previously Invisible Biomarkers. J. Am. Chem. Soc 2011, 133 (47), 19178-19188. [PubMed: 21999289]

7). Magni R; Espina BH; Shah K; Lepene B; Mayuga C; Douglas TA; Espina V; Rucker S; Dunlap R; Petricoin EFI; Kilavos MF; Poretz DM; Irwin GR; Shor SM; Liotta LA; Luchini A Application of Nanotrap Technology for High Sensitivity Measurement of Urinary Outer Surface Protein A Carboxyl-Terminus Domain in Early Stage Lyme Borreliosis. J. Transl. Med 2015, 13 (1), 346. [PubMed: 26537892]

8). Byrne ME; Oral E; Zachary Hilt J; Peppas NA Networks for Recognition of Biomolecules: Molecular Imprinting and Micropatterning Poly(ethylene Glycol)- Containing Films. Polym. Adv. Technol 2002, 13 (10-12), 798-816.

9). Kryscio DR; Peppas NA Critical Review and Perspective of Macromolecularly Imprinted Polymers. Acta Biomater. 2012, 8 (2), 461-473. [PubMed: 22100344]

10). Zhang Z; Liu J Molecularly Imprinted Polymers with DNA Aptamer Fragments as Macromonomers. ACS Appl. Mater. Interfaces 2016, 8 (10), 6371-6378. [PubMed: 26910515]

11). Hermanson G Bioconjugate Techniques, 2nd ed.; Academic Press: Boston, 2008.

12). Yan L; Zhu Z; Zou Y; Huang Y; Liu D; Jia S; Xu D; Wu M; Zhou Y; Zhou S; Yang CJ TargetResponsive "Sweet" Hydrogel with Glucometer Readout for Portable and Quantitative Detection of Non-Glucose Targets. J. Am. Chem. Soc 2013, 135 (10), 3748-3751. [PubMed: 23339662]

13). Kiyonaka S; Sada K; Yoshimura I; Shinkai S; Kato N; Hamachi I Semi-Wet Peptide/protein Array Using Supramolecular Hydrogel. Nat. Mater 2004, 3 (1), 58-64. [PubMed: 14661016]

14). Knipe JM; Chen F; Peppas NA Enzymatic Biodegradation of Hydrogels for Protein Delivery Targeted to the Small Intestine. Biomacromolecules 2015, 16 (3), 962-972. [PubMed: 25674922]

15). Anderson SB; Lin C-C; Kuntzler DV; Anseth KS The Performance of Human Mesenchymal Stem Cells Encapsulated in CellDegradable Polymer-Peptide Hydrogels. Biomaterials 2011, 32 (14), 3564-3574. [PubMed: 21334063]

16). Brubaker CE; Messersmith PB Enzymatically Degradable Mussel-Inspired Adhesive Hydrogel. Biomacromolecules 2011, 12 (12), 4326-4334. [PubMed: 22059927]

17). Cai Z; Smith NL; Zhang J-T; Asher SA Two-Dimensional Photonic Crystal Chemical and Biomolecular Sensors. Anal. Chem 2015, 87 (10), 5013-5025. [PubMed: 25867803]

18). Zhang J-T; Wang L; Luo J; Tikhonov A; Kornienko N; Asher SA 2-D Array Photonic Crystal Sensing Motif. J. Am. Chem. Soc 2011, 133 (24), 9152-9155. [PubMed: 21604702]

19). Ben-Moshe M; Alexeev VL; Asher SA Fast Responsive Crystalline Colloidal Array Photonic Crystal Glucose Sensors. Anal. Chem 2006, 78 (14), 5149-5157. [PubMed: 16841941]

20). Zhang J-T; Cai Z; Kwak DH; Liu X; Asher SA TwoDimensional Photonic Crystal Sensors for Visual Detection of Lectin Concanavalin A. Anal. Chem 2014, 86 (18), 9036-9041. [PubMed: 25162117]

21). Armstrong E; O’Dwyer C Artificial Opal Photonic Crystals and Inverse Opal Structures Fundamentals and Applications from Optics to Energy Storage. J. Mater. Chem. C 2015, 3 (24), 6109-6143.

22). Couturier J-P; Sütterlin, M; Laschewsky, A; Hettrich, C; Wischerhoff E Responsive Inverse Opal Hydrogels for the Sensing of Macromolecules. Angew. Chem., Int. Ed 2015, 54 (22), 6641-6644.

23). Hou J; Zhang H; Yang Q; Li M; Jiang L; Song Y Hydrophilic-Hydrophobic Patterned Molecularly Imprinted Photonic Crystal Sensors for High-Sensitive Colorimetric Detection of Tetracycline. Small 2015, 11 (23), 2738-2742. [PubMed: 25649896] 
24). Hu X; Li G; Huang J; Zhang D; Qiu Y Construction of SelfReporting Specific Chemical Sensors with High Sensitivity. Adv. Mater 2007, 19 (24), 4327-4332.

25). Mesch M; Zhang C; Braun PV; Giessen H Functionalized Hydrogel on Plasmonic Nanoantennas for Noninvasive Glucose Sensing. ACS Photonics 2015, 2 (4), 475-480.

26). Derazshamshir A; Baydemir G; Andac M; Say R; Galaev IY; Denizli A Molecularly Imprinted PHEMA-Based Cryogel for Depletion of Hemoglobin from Human Blood. Macromol. Chem. Phys 2010, 211 (6), 657-668.

27). Baeissa A; Dave N; Smith BD; Liu J DNA-Functionalized Monolithic Hydrogels and Gold Nanoparticles for Colorimetric DNA Detection. ACS Appl. Mater. Interfaces 2010, 2 (12), 35943600. [PubMed: 21077647]

28). Helwa Y; Dave N; Froidevaux R; Samadi A; Liu J AptamerFunctionalized Hydrogel Microparticles for Fast Visual Detection of Mercury(II) and Adenosine. ACS Appl. Mater. Interfaces 2012, 4 (4), 2228-2233. [PubMed: 22468717]

29). King PJS; Saiani A; Bichenkova EV; Miller AF A de Novo Self-Assembling Peptide Hydrogel Biosensor with Covalently Immobilised DNA-Recognising Motifs. Chem. Commun 2016, 52 (40), 6697-6700.

30). Yuan W; Yang J; Kopeckovǎ P; Kopeć ek J Smart Hydrogelš Containing Adenylate Kinase: Translating Substrate Recognition into Macroscopic Motion. J. Am. Chem. Soc 2008, 130 (47), 15760-15761. [PubMed: 18980321]

31). Hoshino Y; Koide H; Furuya K; Haberaecker WW; Lee SH; Kodama T; Kanazawa H; Oku N; Shea KJ The Rational Design of a Synthetic Polymer Nanoparticle That Neutralizes a Toxic Peptide in Vivo. Proc. Natl. Acad. Sci. U. S. A 2012, 109 (1), 33-38. [PubMed: 22198772]

32). Elsabahy M; Wooley KL Design of Polymeric Nanoparticles for Biomedical Delivery Applications. Chem. Soc. Rev. 2012, 41 (7), 2545-2561. [PubMed: 22334259]

33). Hiratani H; Fujiwara A; Tamiya Y; Mizutani Y; AlvarezLorenzo C Ocular Release of Timolol from Molecularly Imprinted Soft Contact Lenses. Biomaterials 2005, 26 (11), 1293-1298. [PubMed: 15475059]

34). Alvarez-Lorenzo C; Yanez F; Barreiro-Iglesias R; Concheiro A Imprinted Soft Contact Lenses as Norfloxacin Delivery Systems. J. Controlled Release 2006, 113 (3), 236-244.

35). White CJ; McBride MK; Pate KM; Tieppo A; Byrne ME Extended Release of High Molecular Weight Hydroxypropyl Methylcellulose from Molecularly Imprinted, Extended Wear Silicone Hydrogel Contact Lenses. Biomaterials 2011, 32 (24), 5698-5705. [PubMed: 21601274]

36). Pan G; Guo Q; Cao C; Yang H; Li B Thermo-Responsive Molecularly Imprinted Nanogels for Specific Recognition and Controlled Release of Proteins. Soft Matter 2013, 9 (14), 3840-3850.

37). Zhang Y; Deng C; Liu S; Wu J; Chen Z; Li C; Lu W Active Targeting of Tumors through Conformational Epitope Imprinting. Angew. Chem., Int. Ed 2015, 54 (17), 5157-5160.

38). Shen X; Svensson Bonde J; Kamra T; Bülow L; Leo JC; Linke D; Ye L Bacterial Imprinting at Pickering Emulsion Interfaces. Angew. Chem., Int. Ed 2014, 53 (40), 10687-10690.

39). Soontornworajit B; Zhou J; Shaw MT; Fan T-H; Wang Y Hydrogel Functionalization with DNA Aptamers for Sustained PDGFBB Release. Chem. Commun 2010, 46 (11), 1857-1859.

40). Battig MR; Soontornworajit B; Wang Y Programmable Release of Multiple Protein Drugs from Aptamer-Functionalized Hydrogels via Nucleic Acid Hybridization. J. Am. Chem. Soc 2012, 134 (30), 12410-12413. [PubMed: 22816442]

41). Miyata T; Asami N; Uragami T A Reversibly AntigenResponsive Hydrogel. Nature 1999, 399 (6738), 766-769. [PubMed: 10391240]

42). Podual K; Doyle FJ; Peppas NA Glucose-Sensitivity of Glucose Oxidase-Containing Cationic Copolymer Hydrogels Having Poly (ethylene Glycol) Grafts. J. Controlled Release 2000, 67 (1), 9-17.

43). Marek SR; Peppas NA Insulin Release Dynamics from Poly (diethylaminoethyl Methacrylate) Hydrogel Systems. AIChE J. 2013, 59 (10), 3578-3585. [PubMed: 24634515]

44). Gu Z; Aimetti AA; Wang Q; Dang TT; Zhang Y; Veiseh O; Cheng H; Langer RS; Anderson DG Injectable Nano-Network for Glucose-Mediated Insulin Delivery. ACS Nano 2013, 7 (5), 4194 4201. [PubMed: 23638642] 
45). Knipe JM; Strong LE; Peppas NA Enzyme-and pHResponsive Microencapsulated Nanogels for Oral Delivery of siRNA to Induce TNF-A Knockdown in the Intestine. Biomacromolecules 2016, 17 (3), 788-797. [PubMed: 26813877]

46). Gu Z; Yan M; Hu B; Joo K-I; Biswas A; Huang Y; Lu Y; Wang P; Tang Y Protein Nanocapsule Weaved with Enzymatically Degradable Polymeric Network. Nano Lett. 2009, 9 (12), 4533 4538. [PubMed: 19995089]

47). Byrne JD; Betancourt T; Brannon-Peppas L Active Targeting Schemes for Nanoparticle Systems in Cancer Therapeutics. Adv. Drug Delivery Rev 2008, 60 (15), 1615-1626.

48). Nicolas J; Mura S; Brambilla D; Mackiewicz N; Couvreur P Design, Functionalization Strategies and Biomedical Applications of Targeted Biodegradable/biocompatible Polymer-Based Nanocarriers for Drug Delivery. Chem. Soc. Rev. 2013, 42 (3), 1147-1235. [PubMed: 23238558]

49). Xu X; Ho W; Zhang X; Bertrand N; Farokhzad O Cancer Nanomedicine: From Targeted Delivery to Combination Therapy. Trends Mol. Med 2015, 21 (4), 223-232. [PubMed: 25656384] 
SENSORS

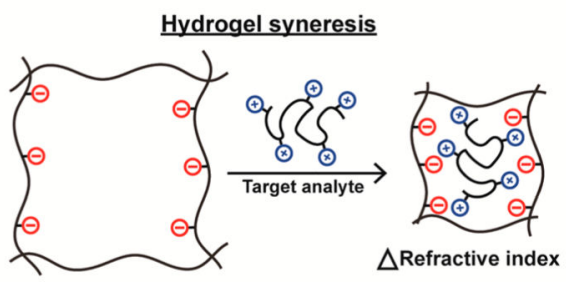

Hydrogel assembly

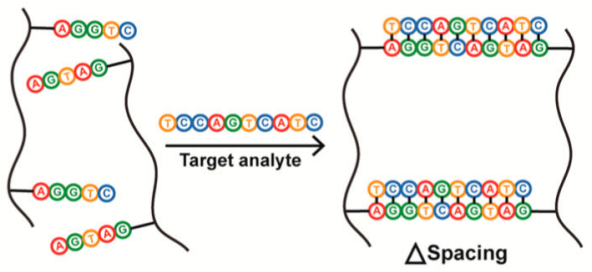

Molecular reorientation

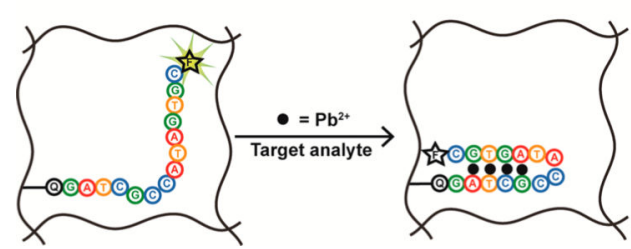

DRUG DELIVERY SYSTEMS

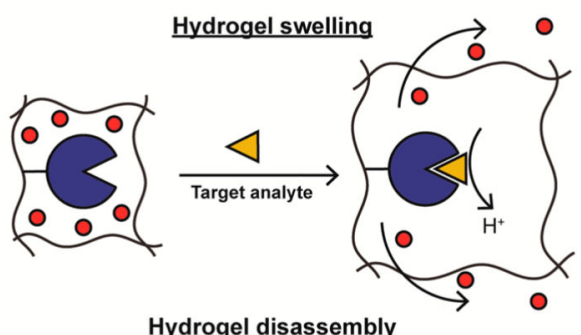

Hydrogel disassembly

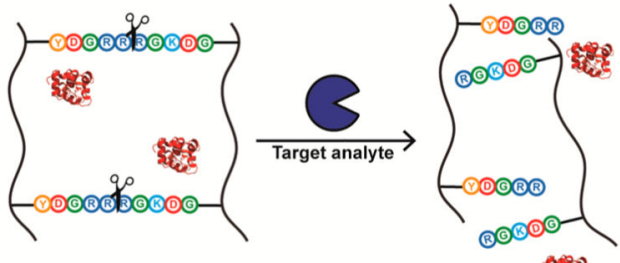

दैध

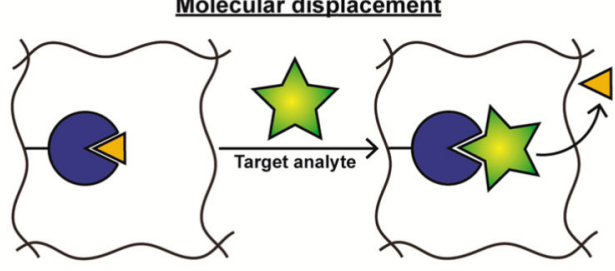

Figure 1.

Hydrogels can be designed to respond to diverse analytes using a variety of molecular recognition approaches. Furthermore, the interaction between the target analyte and hydrogel result in conformational changes that can be exploited for sensing and drug delivery. 
(a)

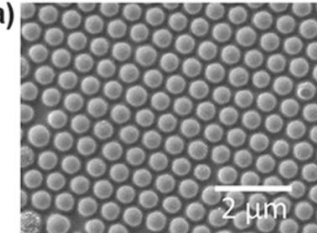

(b)

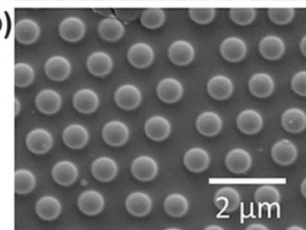

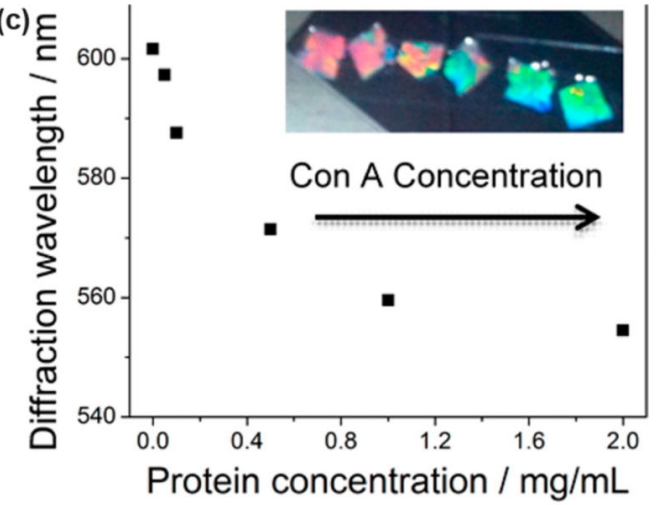

Figure 2.

SEM images of a 2D array of polystyrene nanoparticles with mannose-hydrogel-filled interstices showing (a) a close-packed array when the hydrogel is collapsed and (b) a nonclose-packed array when the hydrogel is swollen. (c) Dependence of the normalized diffraction spectra of 2D mannose hydrogel sensors on the concanavalin A concentration. The inset photographs demonstrate the sensor's color change with increasing concanavalin A concentration. Reprinted from ref 20. Copyright 2014 American Chemical Society. 

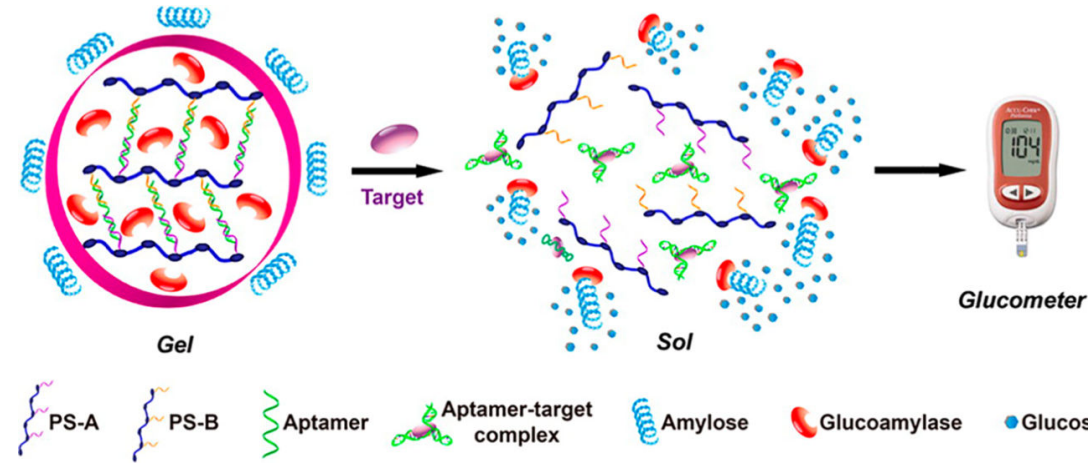

Aptamer-target

Sol

$\because$

Glucometer

Figure 3.

An aptamer-cross-linked hydrogel with trapped glucoamylase is formed by hybridization of the aptamer and its partially complementary DNA polymer strands (PS-A and PS-B). When target molecules are introduced, the aptamers specifically identify the targets to form target -aptamer complexes, causing breakdown of the hydrogel and release of glucoamylase, which catalyzes the hydrolysis of amylose to produce a large amount of glucose for quantitative readout by the glucometer. Reprinted from ref 12. Copyright 2013 American Chemical Society. 


\begin{tabular}{|c|c|c|}
\hline & Competitive System & Physiochemical Modification \\
\hline $\begin{array}{l}\frac{0}{0} \\
\frac{\pi}{0} \\
\frac{\pi}{0} \\
\text { 0. } \\
0\end{array}$ & $\begin{array}{l}\text { Mechanism: } \\
\downarrow \text { Crosslinking } \\
\text { Examples: } \\
\text { Antibody-Antigen Gels } \\
\text { Disulfide Crosslinker- } \\
\text { Containing Gels }\end{array}$ & $\begin{array}{l}\text { Mechanism: } \\
\downarrow \text { Crosslinking } \\
\text { Examples: } \\
\text { Enzymatically-Degradable Gels } \\
\text { pH-Labile Crosslinker- } \\
\text { Containing Gels }\end{array}$ \\
\hline $\begin{array}{l}\frac{0}{0} \\
\frac{0}{0} \\
\frac{\pi}{00} \\
0 \\
0 \\
0 \\
0 \\
0 \\
2\end{array}$ & $\begin{array}{l}\text { Mechanism: } \\
\text { Present Analyte Abundant } \\
\text { Retention } \\
\text { Examples: } \\
\text { Recognitive Polymers } \\
\text { Molecularly Imprinted Polymers } \\
\text { Ligand-Conjugated Gels }\end{array}$ & $\begin{array}{c}\text { Mechanism: } \\
\text { Analyte } \\
\text { Examples: } \\
\text { EH-Responsive } \\
\text { Enzyme-Conjugated Gels }\end{array}$ \\
\hline
\end{tabular}

Figure 4.

Mechanisms underlying common responsive hydrogel drug delivery systems, categorized by analyte-drug competition and the destructive or nondestructive hydrogel response. 
A<smiles>C=CC(=O)NC</smiles>

BIS:

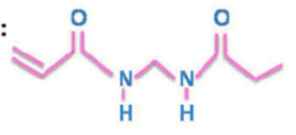

p32: $\quad$ TDGDKAFVDFLS

Apamin:

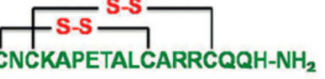

Template:

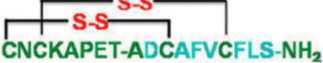

C

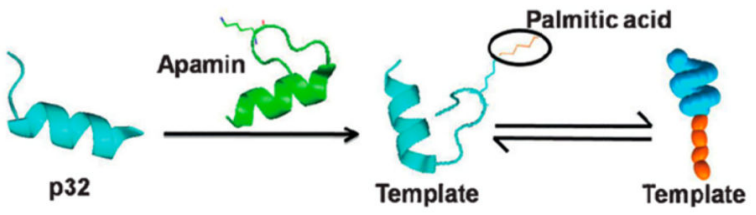

Functional monomers

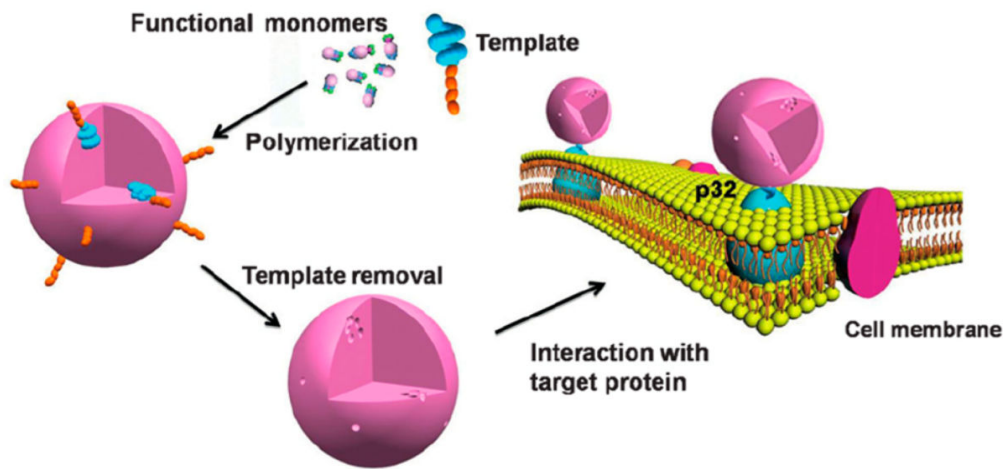

Figure 5.

Epitope-imprinted polymer nanoparticles specifically recognized the p32 receptor, were taken up by $\mathrm{p} 32$-expressing tumors, and delivered a small-molecule photosensitizer. Reprinted with permission from ref 37. Copyright 2015 John Wiley and Sons. 
a)

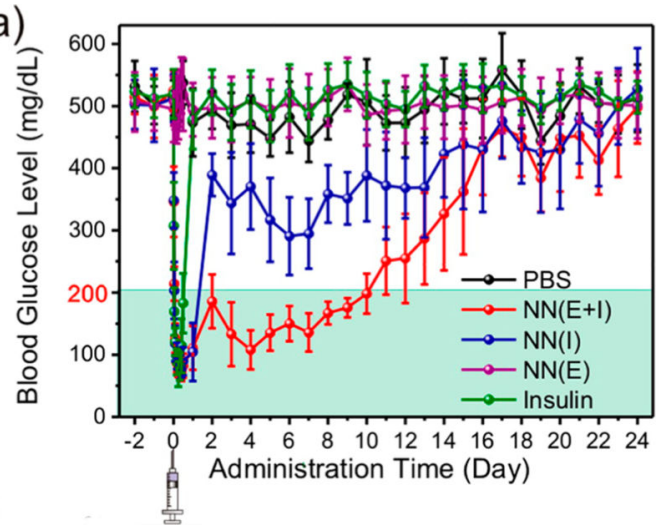

b)

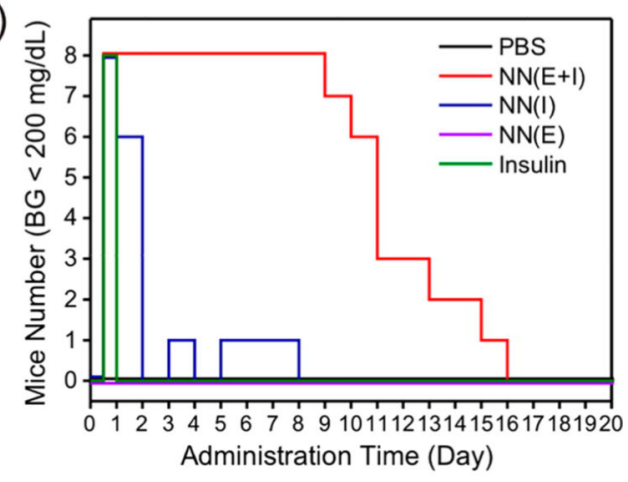

Figure 6.

(a) Diabetic mice injected with insulin-loaded glucose-responsive nanonetworks sustained normoglycemic levels, whereas mice receiving a bolus insulin dose or control treatments became rapidly hyperglycemic. (b) Normoglycemia duration for mice from each treatment group, following treatment. Reprinted from ref 44. Copyright 2013 American Chemical Society. 